\title{
Archéopages
}

Archéopages Archéologie et société

43 | 2016

Médecines

\section{Le verre dans la sphère médicale}

Une utilisation plurielle de l'Antiquité au Moyen Âge

Glass in the medical sphere. A plural use from Antiquity to the Middle Ages

El vidrio en la esfera médica. Un uso plural desde la Antigüedad hasta la Edad

Media

Lydie Joan

\section{OpenEdition}

Journals

Édition électronique

URL : https://journals.openedition.org/archeopages/1975

DOI : 10.4000/archeopages. 1975

ISSN : 2269-9872

Éditeur

INRAP - Institut national de recherches archéologiques préventives

Édition imprimée

Date de publication : 1 novembre 2016

Pagination : 44-51

ISSN : 1622-8545

\section{Référence électronique}

Lydie Joan, «Le verre dans la sphère médicale », Archéopages [En ligne], 43 | 2016, mis en ligne le 01 décembre 2018, consulté le 03 juin 2021. URL : http://journals.openedition.org/archeopages/1975 ; DOI : https://doi.org/10.4000/archeopages. 1975 


\section{Le verre dans la sphère médicale Une utilisation plurielle de l'Antiquité au Moyen Âge}

Lydie Joan Irrap

44

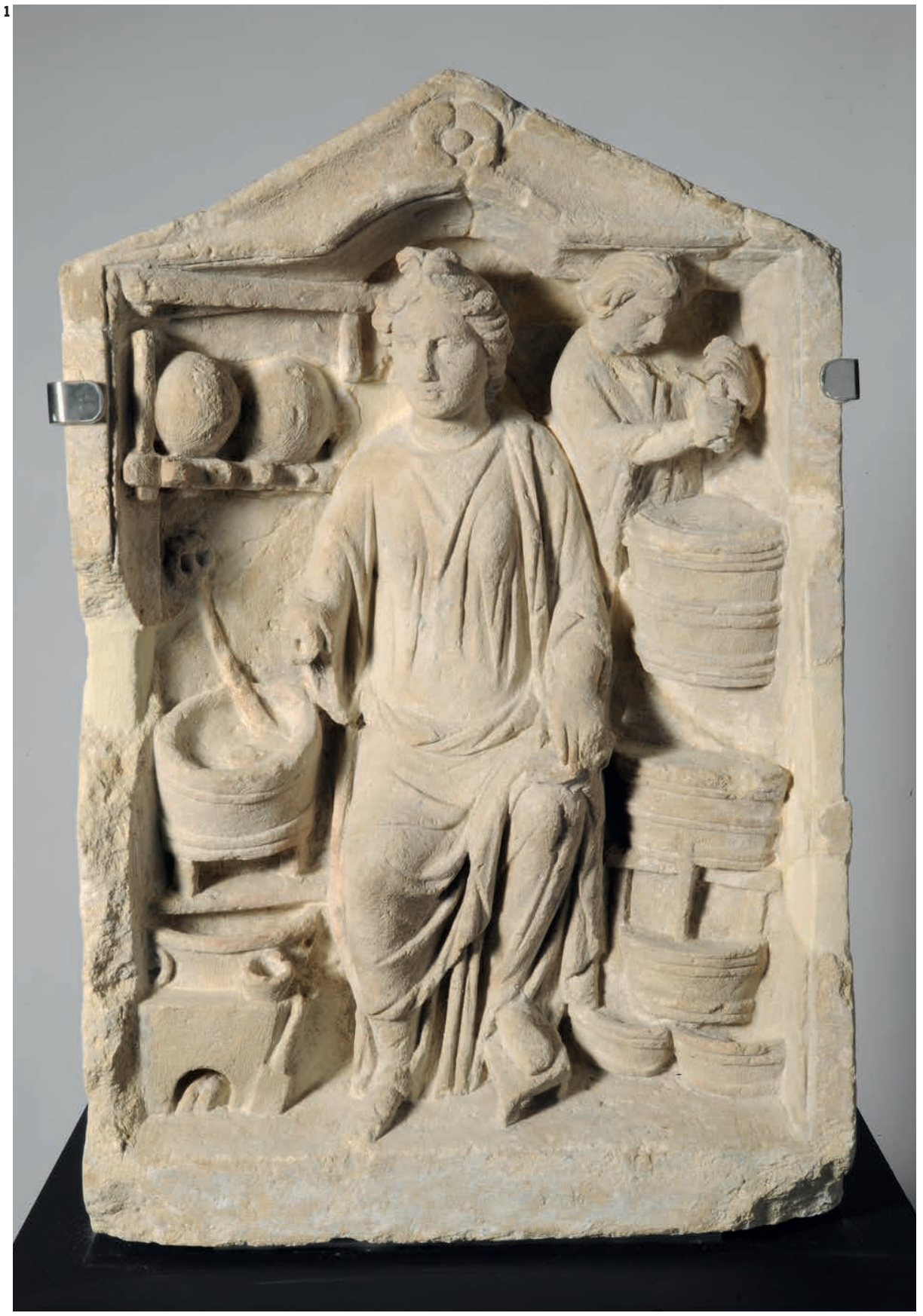


La fragilité de cette matière et son recyclage, pratiqué dès l'Antiquité, font que de nombreux objets ont à jamais disparu, brisés et/ou refondus. Ceux qui parviennent jusqu'aux mains des archéologues sont issus de contextes funéraires ou domestiques. Or ces environnements ne mettent que rarement en lumière le lien potentiel de ces verreries avec la sphère médicale.

Face à la variété typologique des balsamaires, des fioles, des flacons et autres bouteilles en verre, l'archéologue est aussi fort démuni. En effet, en l'absence d'étiquettes au titre évocateur, comment déterminer les contenus de ces récipients et ainsi identifier ceux ayant trait aux soins? Seul, le récipient ne peut donc restituer, dans une juste mesure, l'emploi de cette matière dans le domaine thérapeutique. Face à ces limites, sources écrites, iconographie et chimie permettent cependant d'appréhender les rapports entre ce matériau et cette discipline, corrélation confirmée par quelques découvertes archéologiques exceptionnelles.

\section{Les sources écrites, l'iconographie et la chimie au secours de l'archéologie?}

Des textes antiques et médiévaux évoquent ou sous-entendent l'utilisation du verre en médecine : traités médicaux ou naturalistes, inventaires d'officines... Pour la période antique, on peut citer des écrits de médecins tel Hippocrate, ou encore ceux de naturalistes comme Pline l'Ancien (Histoire naturelle). Bien que les références soient discrètes, elles associent à la médecine des objets en cristal ou en verre. Ainsi le meilleur cautère est, pour Pline l'Ancien, une boule de cristal recevant les rayons du soleil (Histoire naturelle, XXXVII, $37,2)$. Ce même auteur recommande clairement, dans certaines recettes médicinales, la macération au soleil de certains ingrédients dans des vases de verre (Histoire naturelle, XXI, 73, 1).

À ces textes, on peut confronter l'image. Quelques reliefs antiques illustrent le monde thérapeutique. Mais il est souvent difficile de déterminer la matière même des objets ou des récipients représentés. Si le symbole du médecin dans l'iconographie antique est la ventouse, comment déterminer s'il s'agit de modèles en bronze ou en verre ? Les deux matières ont, en effet, été utilisées pour la fabrication de ces objets. Parfois, c'est l'interprétation même de la scène qui est sujette à discussion. Ainsi, la représentation de bouteille ou de ballon en verre est parfois envisagée sur la stèle dite de Meditrina, déesse des médecins et des remèdes (Grand, Vosges, II ${ }^{\mathrm{e}}$ siècle) [ill. 1]. Or la divinité représentée peut aussi être interprétée comme une Abondance dispensant ses bienfaits à d'autres artisanats comme la savonnerie, la verrerie ou encore la teinturerie...

C'est en fait à la période médiévale que le verre est formellement identifiable dans les scènes aux thèmes thérapeutiques illustrées dans les enluminures, les peintures et les vitraux. À cette époque, le médecin est souvent représenté un flacon de verre à la main. Il s'agit de la matula, récipient destiné à l'uroscopie, aux formes et proportions variées (Moulinier-Brogi, 2012). Elle devient l'emblème de saint Côme, patron des médecins alors que le pot à onguent, souvent en bois ou en os, est celui de saint Damien, patron des pharmaciens. Toutefois, chez ces derniers, les archives, tels les inventaires d'officine, confirment bien une large utilisation des bouteilles et des flacons dans les pharmacies de la fin du Moyen Âge.

Enfin, les analyses chimiques des résidus organiques permettent de nous instruire sur les contenus de certains récipients en verre. Depuis les années 1990, plusieurs équipes composées de chimistes et d'archéobotanistes se sont spécialisées dans l'identification des marqueurs chimiques des matériaux biologiques dégradés (Garnier, 2012). Les données archéométriques aident ainsi à retrouver certains composants entrant dans les recettes de remèdes. Comme la pharmacopée ancienne est bien connue au travers de nombreux textes antiques - notamment ceux de Théophraste, Celse, Pline l'Ancien (Recherches sur les plantes; Traité de médecine de A.C. Celse ; Histoire naturelle) - ou de manuscrits médiévaux (Antidotarium Magnum...), l'identification de récipients en verre utilisés dans le monde médical devient alors possible. Mais l'exercice demeure toutefois périlleux pour l'Antiquité, car les principes actifs des parfums, baumes et onguents avaient une valeur cosmétique aussi bien que thérapeutique.

\section{Une matière aux caractéristiques physiques adaptées aux soins}

Malgré sa fragilité, le verre se révèle une matière appréciée des divers acteurs de la médecine antique ou médiévale, et ce, en raison de certaines de ces qualités physiques : transparence, colorisation, neutralité chimique et olfactive, capacité optique.

La première de ses caractéristiques est la transparence. Quand le verre est incolore, cette dernière facilite notamment l'observation de liquides tels que l'urine ou le lait. Ainsi, lors de ses diagnostics, Hippocrate ( $\mathrm{v}^{\mathrm{e}}$ siècle avant notre ère) pratique l'examen de la couleur des urines dans un vase dénommé matula (vase de nuit). Ce type d'expertise se répand au Moyen Âge. Ce type de flacon identifiable dans la typologie des verres des $\mathrm{XIV}^{\mathrm{e}}$ et $\mathrm{XV}^{\mathrm{e}}$ siècles est largement illustré par de nombreuses figurations médiévales [ill. 2]. Dans son traité sur les maladies des femmes, Soranos d'Ephèse, médecin à Rome, sous Trajan et Hadrien, atteste, quant à lui, de l'usage de la tétine artificielle et de l'importance de l'examen du lait. Frédéric Loridant et Nadine Rouquet envisagent même que l'opacité de la terre cuite, incommode pour ces examens, expliquerait l'expansion du biberon 
2. Dessins des formes

d'urinaux d'après les trois

documents suivants:

a. enluminure extraite de

l'ouvrage « Des propriétés

des choses I) de Barthélemy

I'Anglais, Le Mans, France,

$x v^{e}$ siècle;

b. miniature extraite

du « Recueil des Traités

de médecine » de Gérard

de Crémone, 1250-1260

(AI-Razi tenant une matula);

C. enluminure extraite

de l'ouvrage les ur Grandes

heures d'Anne de Bretagne "I,

xvl ${ }^{e}$ siècle (Saint Côme

et Saint Damien).
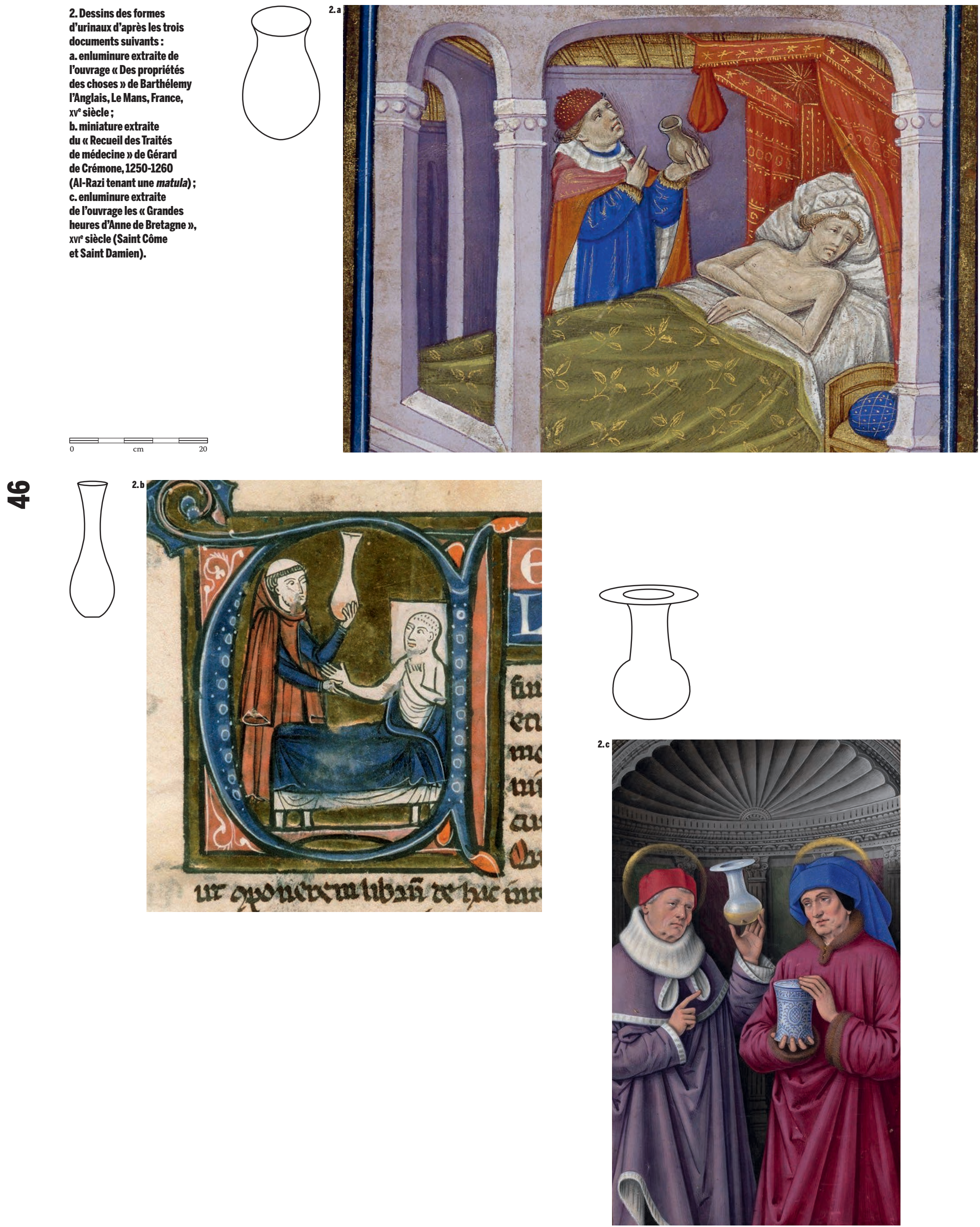
de verre dès le $\mathrm{II}^{\mathrm{e}}$ siècle en Gaule pour devenir quasi-exclusif aux IV $\mathrm{V}^{\mathrm{e}}$ et $\mathrm{V}^{\mathrm{e}}$ siècles (Rouquet, Loridant, 200o, p. 428-431).

A contrario, quand il n'est pas incolore, le verre est apprécié pour sa large gamme de couleurs ; il peut se substituer symboliquement à certaines pierres, parées de vertus guérisseuses ou préventives durant l'Antiquité. Ainsi, des intailles en pâte de verre rouge sont utilisées à la place de la cornaline ou du jaspe dont le pouvoir présumé était de réguler les flux sanguins et de stopper les hémorragies. Les propriétés de ces pierres précieuses ou verroteries étaient souvent renforcées par la représentation gravée de divinités comme Hercule. De telles amulettes ou gemmes en verre coloré ont continué d'être en usage au Moyen Âge (Cannella, 2006).

La troisième propriété du verre est sa neutralité chimique, idéale pour la préparation des médicaments et des cosmétiques ou la conservation longue de liquides aux vertus médicinales : collyre, onguents, baume... Pline l'Ancien recommande clairement, dans son Histoire naturelle, de conserver dans des vases de verre l'urine de sanglier qu'il vante dans le traitement des douleurs d'oreilles (Histoire naturelle, XXVIII, 48, 1). Au-delà de la conservation, les alchimistes égyptiens Bolos de Mendes ( $\mathrm{II}^{\mathrm{e}}$ siècle avant notre ère) et Marie la Juive (vers le $\mathrm{I}^{\mathrm{er}}$ siècle de notre ère) reconnaissent les qualités du verre pour manipuler des substances très corrosives comme le mercure. Marie la Juive est ainsi à l'origine d'une invention qui, au cours des siècles suivants, va devenir l'un des instruments les plus courants dans les laboratoires de chimie : le trilikos, un alambic destiné à la distillation relié par des tubes à trois récipients en verre. Il est fort probable que ce type d'alambic a aussi été utilisé pour la distillation de plantes pour la confection de recettes médicinales.

Inodore, le verre permet de conserver, sans les altérer, les senteurs considérées comme thérapeutiques dès l'époque antique. Si le procédé utilisé dans l'Antiquité est la macération des plantes, la distillation inventée au Moyen Âge va être à l'origine de la diffusion des huiles essentielles.

Enfin, au Moyen Âge, la capacité de ce matériau à grossir a permis le développement des verres de vue. Dès Aristote, les principaux défauts de la vue deviennent un sujet de réflexion, non seulement chez les médecins, mais aussi chez les philosophes et les physiciens. Au $\mathrm{I}^{\mathrm{er}}$ siècle, Sénèque constate ainsi qu'un objet observé à travers un ballon de verre rempli d'eau apparaît plus gros. Pline l'Ancien rapporte que Néron regardait les combats de gladiateurs à travers une émeraude, qui peut être interprétée comme une lentille de vue (Histoire naturelle, XXVII, 16, 2). Si un exemplaire de lentille grossissante date du I ${ }^{\text {er }}$ siècle (Beretta, Di Pasquale, 2006) ${ }^{\mathbf{1}}$, c'est au Moyen Âge que se répand la pierre de lecture, loupe grossissante posée sur les écrits. Si les premiers exemplaires ( $\mathrm{IX}^{\mathrm{e}}$ siècle) sont en pierre semi-précieuse comme le cristal de roche, il faudra attendre une meilleure qualité du verre qui présente encore trop de bulles et d'impuretés pour être employé à cette fin. C'est au XIII ${ }^{\mathrm{e}}$ siècle que le verre va être utilisé pour les loupes et surtout les premières lunettes. On peut ainsi se référer aux représentations de lunettes (les bésicles) sur une fresque de Tommaso de Modena (1352) ou un tableau de Conrad Von Soest (1404).

De manière plus anecdotique, le caractère incisif du verre le fait apparaître dans une recette de « dentifrice » figurant dans les Compositiones de Scribonius Largus (Scribonii largi compositiones, 6o). Ainsi son dentifrice de Messaline « qui rend les dents blanches et qu'utilise Messaline, femme de notre divin César » est composé de poudre de roses séchées au soleil mélangées avec du verre blanc pilé. La présence de verre pulvérisé associé à des sangsues est aussi signalée dans une recette médiévale pour les soins du corps (Moulinier-Brogi, 2004).

\section{L'Antiquité romaine, entre médecine, cosmétologie et magie}

La frontière entre remède et parfum est assez floue à l'époque antique. En effet, une valeur thérapeutique est attribuée aux principes actifs des cosmétiques que sont les parfums, onguents et baumes. Ainsi, le suc extrait des pétales de roses est, par exemple, reconnu pour ses propriétés astringentes et rafraichissantes (Pline, Histoire naturelle, XXI, 73). Une huile parfumée à la rose pouvait alors avoir une dimension pharmaceutique aussi bien que cosmétique. La frontière est si ténue que le terme latin d'unguentarii (récipients à parfum), réservé à l'origine aux parfumeurs, s'est étendu, au fil du temps, aux fabricants de médicaments. Outre l'unguentarium, plusieurs termes antiques font référence à des récipients à parfum comme ampulla (petit vase fermé ou fiole à panse globulaire ou lenticulaire, pourvue d'un col étroit et allongé) ou encore guttus (vase calibré pour un écoulement goutte à goutte). Enfin, un terme moderne a été aussi inventé pour qualifier ces récipients : le balsamaire. Dans l'acception moderne, l'unguentarium fait référence à un contenant pour onguent (pommade à base de résine, corps gras) alors que le balsamaire implique la présence d'un baume (préparation aromatique ne contenant pas de résine et possédant un effet sédatif sur la douleur). Mais, en l'absence d'identification du contenu, et face à la typologie variée des flacons découverts en contextes funéraire, domestique ou thermal, le spécialiste du verre peut rarement trancher. En Gaule romaine, outre les balsamaires et autres unguentarii, pour compléter la liste des récipients en verre voués aux cosmétiques, on peut aussi citer l'aryballe, l'amphorisque, les flacons plats, cylindriques, sphériques ou à panse bulbeuse, ou en grappe, et certains petits pots qu'ils soient piriformes, à
1. La plus ancienne lentille, dite de Nimrud, est en cristal de roche. Découverte à Kalkhu, avant notre ère. Sa fonction exacte demeure inconnue. 
$2^{\mathrm{e}}$ moitié du I $^{\mathrm{e}}$ siècle.

$\mathbb{8}$
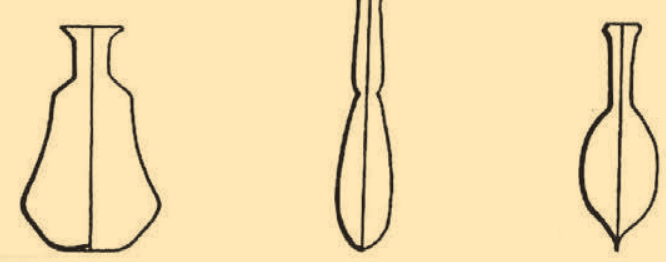

c. À partir du II siècle.

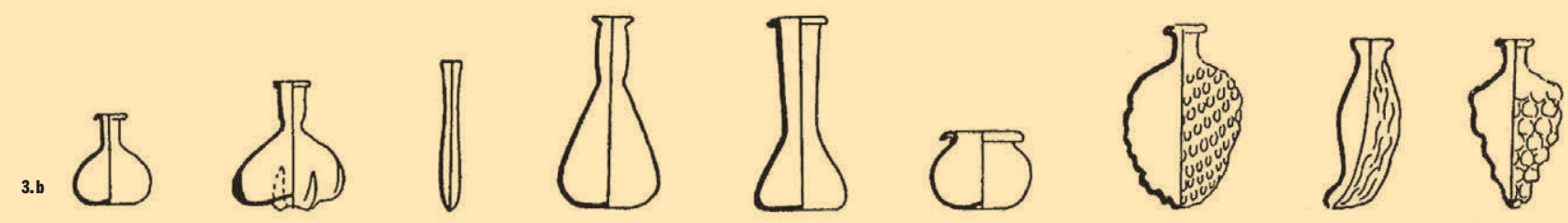

$\stackrel{\infty}{+}$
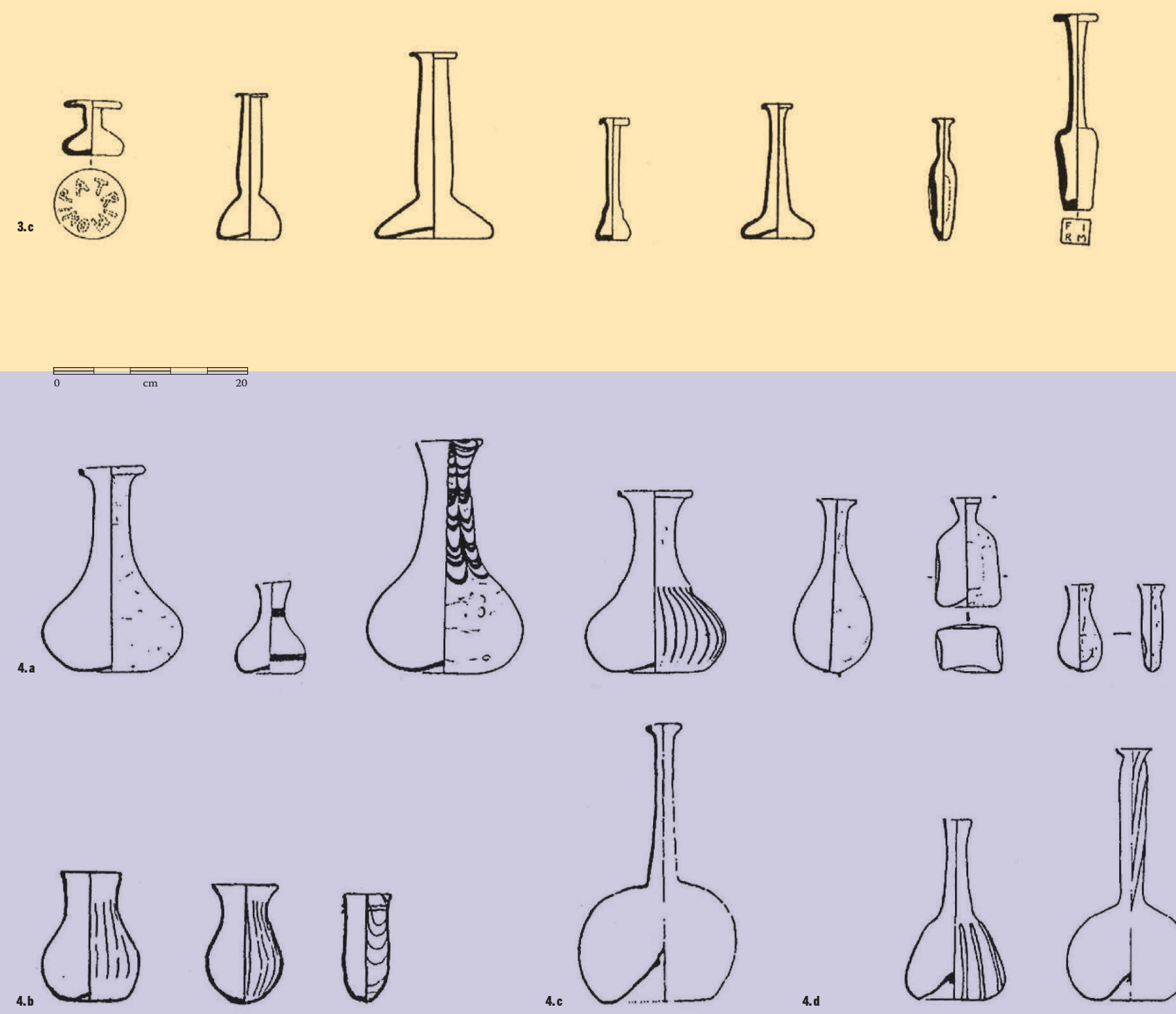

4.d
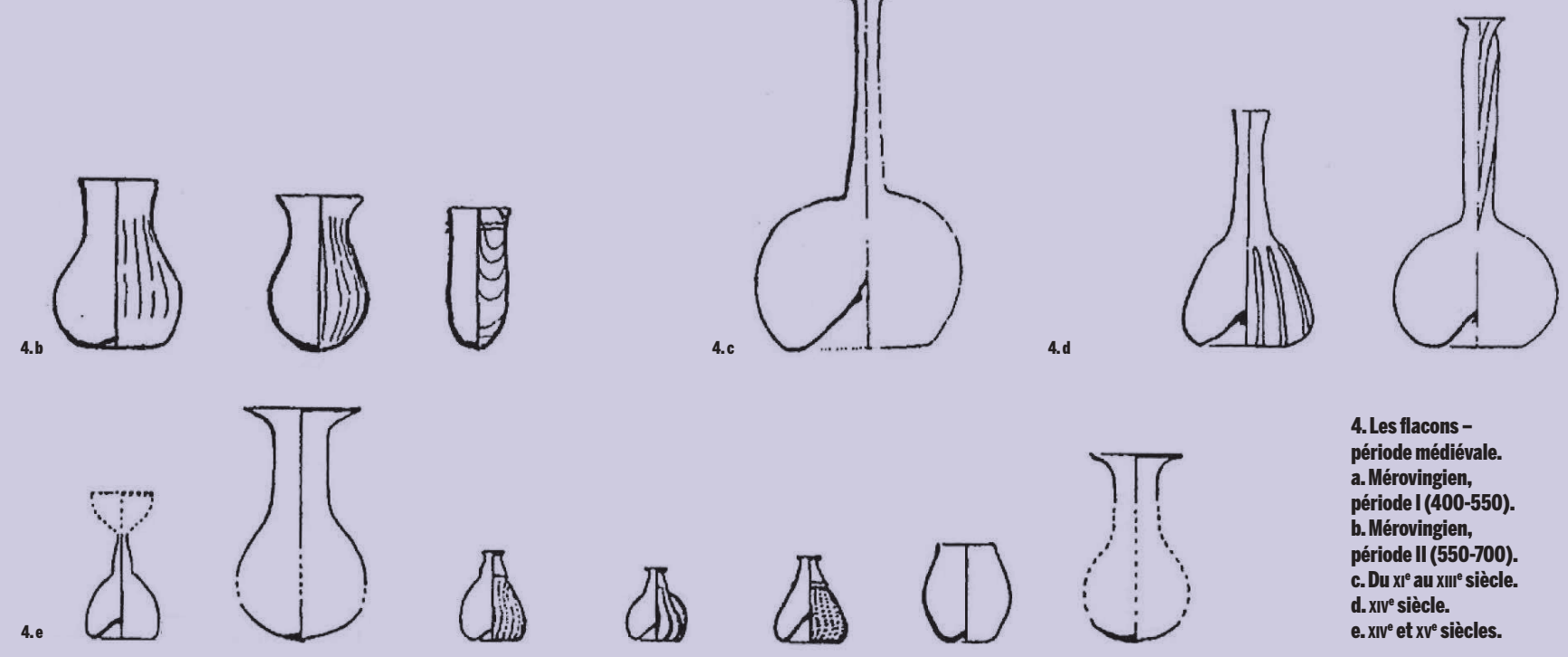

4. Les flacons -

période médiévale

a. Mérovingien,

période I (400-550).

b. Mérovingien,

période II (550-700).

c. Du XI au XIII' siècle.

d. XIve siècle.

e. XIV' et Xve siècles. 
dépressions, ou à onguents et dont la typologie fluctue selon les siècles [ill. 3].

Des quatre tombes de médecins identifiées découvertes en Gaule romaine (Musée du Puy ; Musée Carnavalet, Paris ; Musée d'Archéologie Nationale, Saint-Germain-en-Laye), c'est celle de Saint-Médard-des-Prés en Vendée qui nous éclaire sur la part de ces flacons en verre dans la sphère médicale. En effet, cette tombe d'un oculiste aurait livré pas moins de 80 vases en verre dont 28 sont encore conservés au musée de Fontenay-le-Comte (Santrot, Corson, 2012). Outre des pots globulaires ou carrés, des bouteilles hexagonales, carrées ou cylindriques, 33 balsamaires ont été recensés. Des analyses anciennes et récentes ont permis de démontrer la présence, dans certains de ces contenants, de cires, d'huiles ou d'onguents.

Toute cette gamme de flacons était donc utilisée par les médecins (medici) mais aussi par d'autres corps de métiers plus ou moins rigoureux, à la fois guérisseurs, herboristes, apothicaires, droguistes (pharmacopola), fabricants d'onguents, débitants de poudres et parfumeurs (seplasiarii). Car, outre les vertus médicinales des plantes, Pline, dans son Histoire Naturelle, souligne leurs propriétés magiques. Ainsi, ces flacons pouvaient aussi contenir des mélanges plus magiques que curatifs comme des philtres ou encore des onguents apotropaïques.

Parallèlement, parmi les récipients appartenant au domaine de la toilette, il faut évoquer aussi l'agitateur, bâtonnet lisse ou torsadé qui servait à étaler, écraser et mélanger les produits cosmétiques. Plus exceptionnels sont les strigiles miniatures en verre. En 2003, à Nîmes (Gard), une tombe interprétée comme celle d'un guérisseur (Manniez, 2005) datée de la fin du ${ }^{\mathrm{er}}$ siècle de notre ère a livré des vases en céramique et en verre, des coquillages et des petits objets en os, bronze, verre et cristal de roche. Parmi l'instrumentum, se trouvaient deux petits strigiles en verre bleu clair (L. : 84 et $85,5 \mathrm{~mm}$ ). Par leur taille, ces objets pouvaient servir à appliquer ou à retirer en douceur les préparations sur des parties lésées d'un corps ou à distiller des gouttes dans l'oreille comme le recommande Celse, médecin du i ${ }^{\mathrm{er}}$ siècle (Traité de médecine, VI, 7, 1). Cette dernière utilisation expliquerait l'extrémité bouletée qui diffère de ceux en métal, sans doute destinée à ne pas blesser le conduit de l'oreille. Ces strigiles étaient accompagnés de talismans, amulette en cristal de roche, porcelaine provenant de la mer Rouge et d'une fibule à tête de Méduse dont la valeur apotropaïque permettait de se protéger contre les mauvais sorts, voire de la maladie.

Actuellement, le seul objet en verre que l'on relie, sans équivoque, au monde de la médecine, est la ventouse (cucurbita), ce récipient en forme de cloche destiné à soigner en induisant une « révulsion » par effet de succion sur la peau. Pour ce faire, on chauffait la ventouse puis on l'appliquait sur la zone à traiter, de manière à ce qu'en refroidissant, par sa contraction, se produise un puissant effet de succion. Selon la théorie d'Hippocrate, cette « révulsion » permettait aussi de rétablir l'équilibre entre les humeurs (le sang, la bile, la lymphe et l'atrabile) attirées sous la surface de la peau lorsqu'elles sont en excès. Un exemplaire de ventouse en verre est conservé au musée du Louvre (Iran, $\mathrm{I}^{\mathrm{er}}-\mathrm{II}^{\mathrm{e}}$ siècle). En Gaule, ce sont surtout des modèles en bronze qui nous sont parvenus (Martigny, Suisse ; nécropole de Saint-Marcel à Paris).

Enfin, en ce qui concerne les biberons, vases à becs tubulaires, qu'ils soient en céramique ou en verre, ils soulèvent encore des interrogations dans la communauté scientifique, d'autant qu'ils sont présents dans un certain nombre de tombes d'adultes. Certains chercheurs y voient aussi un tire-lait (Rouquet, Loridant, 2003) ou une tasse à malade (Dubois, 2012, p. 337 ; Jaeggi, Wittmann, Garnier, 2015, p. 572). Des analyses ont montré, à plusieurs reprises, la présence de traces de produits laitiers, mais aussi de vin, de miel, etc., et ce, dans un même vase (Jaeggi, Wittmann, Garnier, 2015). Or ces ingrédients entrent dans de nombreuses recettes médicinales. Les analyses ne permettent donc pas de trancher sur la question de la fonction de ces objets. Fonction sûrement multiple puisque Caelius Aurelianus (médecin $\mathrm{du}^{\mathrm{e}}$ siècle) évoque, dans un traité de médecine, l'utilisation de ces « biberons » pour administrer une boisson à un malade (Jaeggi, Wittmann, Garnier, 2015, p. 572). Comme l'a souligné Céline Dubois (Dubois, 2012, p. 337), le bombylios, utilisé pour donner à boire au malade, dans la civilisation grecque, et décrit, dans le corpus hippocratique (Hippocrate, Maladies, III, 16), comme un vase à goulot étroit, pourrait bien correspondre aussi à un biberon. Quoi qu'il en soit, l'utilisation de biberon en verre se prolongerait au-delà de l'époque antique puisqu'un traité de gynécologie $\mathrm{du} \mathrm{XIII}^{\mathrm{e}}$ siècle, Les Infortunes de Dinah, conseille de faire boire l'enfant sevré dans un récipient de verre en forme de téton que l'on appelle nad (Barkaï, 1991, p. 157) 2 .

\section{Le Moyen Âge, entre médecine, religion et alchimie}

Au cours du Moyen Âge, les médecins occidentaux vont parfaire les connaissances médicales héritées de l'Antiquité, soit par des expérimentations qui leur sont propres, soit par des contacts avec le monde arabe. Jusqu'aux $\mathrm{X}^{\mathrm{e}}-\mathrm{XII}^{\mathrm{e}}$ siècles, la médecine est alors essentiellement conventuelle car pratiquée par différents acteurs de la religion chrétienne. Une médecine laïque sérieuse se développe ensuite avec l'apparition des universités de médecine ( $\mathrm{XII}^{\mathrm{e}}$-XIII ${ }^{\mathrm{e}}$ siècle). L'évolution de cette discipline peut être illustrée, dans une certaine mesure, par la transformation ou la création de certains objets en verre. Toutefois cette vision n'est que partielle. En effet, actuellement, les fouilles
2. Nad est traduit par l'auteur par gourde. 
archéologiques en contexte médiéval demeurent encore très exceptionnelles pour la sphère médicale (léproserie, apothicairerie...).

En ce qui concerne l'héritage antique, si la ventouse en verre perdure, c'est désormais la matula $^{3}$ qui devient l'emblème du médecin. Pour ce récipient destiné à l'examen des urines, les formes sont variées si l'on en juge par les différentes représentations sur enluminure. Dans la typologie actuelle des verres du nord de la France des XIV ${ }^{\mathrm{e}}$ et $\mathrm{XV}^{\mathrm{e}}$ siècles, il prend la forme d'un vase ovoïde à fond bombé à large ouverture, forme proche des vases à pharmacie et des ballons de distillerie. Ce sont alors les proportions et l'extrême finesse de la paroi et donc une transparence parfaite qui permettent de le caractériser (Foy, Sennequier, 1989, p. 329-330). Ce type de récipient ne se limite pourtant pas à la fonction d'urinal si l'on en croit les médecins Avicenne ou Jacques Despars qui suggèrent d'utiliser un vase comme l'urinal pour manipuler divers ingrédients de recettes médicinales (Jacquart, 1998).

Quant à la typologie des verres médiévaux actuellement proposée par les archéologues, comparée à celle des verres antiques, on constate une nette réduction dans la diversité du flaconnage [ill. 4]. De même, on voit disparaître de la terminologie l'unguentarium et le balsamarius. Ces phénomènes semblent retranscrire un ralentissement des parfums et autres onguents avec l'expansion du christianisme. Toutefois, parfum et guérison continuent à être associés, mais dans le cadre de la religion chrétienne. Une des saintes huiles (oleum infirmorum) servait ainsi à l'onction des infirmes et des malades. Le lien entre Église et médecine est aussi illustré par un nouveau récipient: l'ampoule de pèlerinage permettant aux pèlerins, depuis la fin de l'Antiquité, de transporter huiles saintes, eaux bénites ou encore miraculeuses. Actuellement, les rares exemplaires en verre, en France, concernent uniquement le littoral méditerranéen et datent au plus tard du v siècle (Foy, 2010). Pour le Moyen Âge, ce sont essentiellement des gourdes aplaties en céramique ou en métal qui nous sont parvenues. Toutefois, il est à noter que des ampoules de pèlerinage, en verre, non datées (médiévales ou modernes) du Musée des antiquités de Rouen ont été étudiées par Jorge Barrera (Barrera, 1990). De même, un atelier de petits flacons en verre utilisés pour recueillir le myron de la tombe de saint Démétrios en verre du $\mathrm{Xv}^{\mathrm{e}}$ siècle a été fouillé à Thessalonique en Grèce (Loverdou-

Tsigarida, 2003, p. 245).

Enfin, les verreries aux fonctions liturgiques, burettes, bouteilles à long col décorées à l'émail de Syrie (musée du Louvre) ou encore fiolesreliquaires telle celle de sainte Catherine (Palerme, dans le trésor de la chapelle palatine, palais des Normands), datées des $\mathrm{XII}^{\mathrm{e}}$-XIII ${ }^{\mathrm{e}}$ siècles, ont pu être aussi utilisées dans un cadre de rituels religieux aux fins thérapeutiques.

Si les petits flacons sont moins diversifiés qu'à l'époque antique, le verre est tout de même présent dans les scènes illustrant les médecins et les apothicaires (par exemple, un vitrail de la vie de saint Nicolas, cathédrale de Chartres ; dessin d'une apothicairerie dans le traité de chirurgie de Roger de Salerne). Cette iconographie met en avant, désormais, les grandes fioles (bouteille au long col étroit), un verre probablement doseur et les bocaux. Si on compare la typologie du verre médiéval domestique avec cette iconographie médicale, certaines formes sont très similaires, ce qui nuancerait l'hypothèse de récipients en verre à fonction uniquement médicinale pour le Moyen Âge.

Deux objets, enfin, inconnus des artefacts antiques, illustrent deux des innovations de la médecine médiévale : les débuts de l'optique et la distillation. Si les problèmes de vue furent évoqués dans l'Antiquité par Aristote dans le problemata, en particulier la myopie et la presbytie, l'invention des lunettes fut bien plus longue à venir. Les premières bésicles apparaissent dans le milieu monastique, au XIII ${ }^{\mathrm{e}}$ siècle, afin que les moines puissent voir de près et recopier les manuscrits sacrés.

La distillation, invention arabe du viII ${ }^{\mathrm{e}}$ siècle, est introduite en Occident avec l'alchimie au $\mathrm{XII}^{\mathrm{e}}$ siècle. Elle nécessite l'utilisation d'un alambic si cette distillation se fait par élévation des vapeurs. Cet appareil est décrit dans des textes alchimiques mais aussi des traités médiévaux de médecine. Il sert surtout à la fabrication de l'eau de rose et de l'alcool utilisés majoritairement par les médecins et les apothicaires. De nombreuses fouilles dans des contextes domestiques datés du XII ${ }^{\mathrm{e}}$ au $\mathrm{XVII}^{\mathrm{e}}$ siècle ont livré ce type d'objets (noyaux de découvertes en Alsace, Franche-Comté et Angleterre) dont les modèles en verre les plus anciens datent de la fin du XIV siècle (Thomas, 2009). L'archéologie montre donc que ces objets sont à associer à une distillation dans un contexte familial, dans des milieux plutôt privilégiés, riches et cultivés. Si son usage médical (Thomas, 2009) n'est pas assuré, il ne peut être écarté pour ceux ayant accès aux écrits et donc à la diffusion des recettes d'apothicaire.

Au terme de cette présentation, une typologie du verre exclusivement voué à la sphère médicale s'avère impossible. À l'époque antique, ce fait s'explique par une discipline aux limites floues, où s'entremêlent cosmétologie, croyance, magie, religion et médecine. Aussi de nombreux récipients sont-ils utilisés indistinctement dans différents domaines. Là, seul le contexte, les associations d'objets et les analyses chimiques peuvent nous éclairer quant à leur lien avec le monde de la médecine. À l'époque médiévale, la difficulté d'identification persiste. La découverte antique, signifie le pot

de chambre, le récipient en verre sert à recueillir l'urine des patients alités (urinal). 
d'objets voués uniquement au monde médical est encore peu illustrée par l'archéologie qui traite essentiellement de lots de verres issus de contextes domestiques et funéraires. C'est seulement à partir du XIv ${ }^{\mathrm{e}}$ siècle (mais surtout au $\mathrm{XV}^{\mathrm{e}}$ et $\mathrm{XVI}^{\mathrm{e}}$ siècle) que se rencontrent des verres de médecine et de pharmacie (urinaux, bocaux, ballon de chimie, alambics, lunettes...)

(Foy, Sennequier, 1989).

Malgré ces difficultés, la mise au jour de certains objets d'exception, les textes, l'iconographie et les analyses chimiques permettent de percevoir le lien entre verre et médecine. Cette perception devrait s'éclairer, dans l'avenir, avec l'évolution des analyses chimiques. Elle permettra alors de progresser dans la connaissance de la médecine à l'instar des travaux de Jean-Pierre Brun et Nicolas Garnier sur les parfums dans l'Antiquité.

\section{Références bibliographiques}

BARKaï R. (éd.), 1991, Les infortunes de Dinah, le livre de la génération, la gynécologie juive au Moyen Âge, Paris, Éditions du Cerf, 300 p.

CANnElla A.-F., 2006, Gemmes, verre coloré, fausses pierres précieuses au Moyen Âge : le quatrième livre du Trésorier de philosophie naturelle des pierres précieuses de Jean d'Outremeuse, Genève, Droz, $475 \mathrm{p}$.

Celse, Traité de médecine de A. C. Celse, traduction nouvelle, avec texte latin..., A. VÉDRÈnEs (éd.), Paris, Masson, 1876, 797 p.

Pline l'Ancien, Histoire naturelle, Livre XXI, Livre XXVIII, Livre XXXVII, J. ANDrÉ, A. ERMout, E. DE SAINTDenis (éd.), Paris, Les Belles Lettres, 2003 (Collection des universités de France. Série latine, 170, 192, 206)

Scribonius Largus, Scribonii largi compositiones, S. Sconocchia (éd.), Leipzig, B. G. Teubner, 1983, $130 \mathrm{p}$.

Théophraste, Recherches sur les plantes, S. Amigues (éd.), Paris, Les Belles Lettres, 1989-2006, 5 t. (Collection des universités de France. Série grecque, $314,324,359,432,446$ ).

BARRERA J., 1990, « La verrerie médiévale et moderne, Collection Thaurin, Musée des Antiquités de Rouen ", Revue archéologique de l'ouest, t. 7 , p. 115-129.

Beretta M., Di Pasquale G., 2006, Le verre dans l'Empire romain (catalogue d'exposition, Cité des sciences et de l'industrie, Paris, janv.-août 2006), Florence, Milan, Giunti, Cité des sciences et de l'industrie, $360 \mathrm{p}$.
Dubois C., 2012, « Des objets pour les bébés ? Le dépôt de mobilier dans les sépultures d'enfants en bas âge du monde grec archaïque et classique », in Hermary A. et Dubois C. (éd.), L'enfant et la mort dans l'Antiquité III, Le matériel associé aux tombes d'enfants, Actes de la table ronde internationale organisée à la maison Méditerranéenne des Sciences de L'homme (MMSH) d'Aix-en-Provence, 20-22 janvier 2011, Arles, Éd. Errance, p. 294-340.

Foy D., 2010, « Souvenirs de pèlerinages dans l’Antiquité tardive : vaisselle, ampoules et breloques de verre découvertes en Narbonnaise ", in Archéologie des rivages méditerranéens 50 ans de recherche (actes du colloque d'Arles 28-30 octobre 2009), Paris, Éd. Errance, Ministère de la culture et de la communication, p. 303-311.

Foy D., SENNEQUier G. (dir.), 1989, À travers le verre : du Moyen Âge à la Renaissance, catalogue de l'exposition, Rouen, Musées et monuments départementaux de la Seine-Maritime, 454 p.

GARNIER N., 2012, «Une histoire des analyses organiques de matériaux organiques archéologiques, de Chevreul à nos jours. Enjeux, potentiels et limites actuels ", in Frère D. et Hugot L. (éd.), Les huiles parfumées en Méditerranée occidentale et en Gaule ( VIII ${ }^{e}$ S. av.-VI $I^{e}$ s. apr. J.-C.), actes du colloque Rome, École Française de Rome (16-18 nov. 2009), Rennes, Presses universitaires de Rennes, p. 63-74.

JACQUART D., 1998, La médecine médiévale dans le cadre parisien, $X I V^{e}-X V^{e}$ siècle, Paris, Fayard, $587 \mathrm{p}$.

JaegGi S., Wittmann A., Garnier N., avec la coll. de FrÈre D., 2015, « Biberon or not Biberon? Les analyses biochimiques de contenus et la question de la fonction de vases gallo-romains communément appelés "biberons" ", in Actes du congrès de la SFECAG, Nyon, p. 561-576.

LOVERDOU-Tsigarida K., 2003, « Thessalonique, Centre de production d'objets d'arts au XIV ${ }^{\mathrm{e}}$ siècle », Dumbarton Oaks Papers, vol. 57, Symposium on Late Byzantine Thessalonike, p. 241-254.

MANNIEZ Y., 2005, « Essai d'interprétation d'un lot de mobilier d'offrande issu d'une tombe nîmoise du $\mathrm{I}^{\mathrm{er}}$ siècle de notre ère $(\mathrm{F})$ ", Instrumentum, $\mathrm{n}^{\circ} 21$, p. 34-36.

Moulinier-Brogi L., 2004, « Esthétique et soins du corps dans les traités médicaux latins à la fin du Moyen Âge ", Mediévales, 46, Éthique et pratiques médicales, p. 55-72.

Moulinier-Brogi L., 2012, L'uroscopie au Moyen Âge: lire dans un verre la nature de l'homme, Paris, Honoré Champion, 256 p.

ROUQUeT N., LORIDANT F., 200o, « Notes sur les biberons en Gaule romaine », in Actes du congrès de la SFECAG, Libourne, p. 425-440.

Rouquet N., LORIDANT F., 2003, "Archéologie expérimentale : les tire-lait à l'épreuve », in Actes du congrès de la SFECAG, Saint-Romain-En-Gal, p. 665-666.

Santrot J., Corson S., avec la coll. de Bernard E., Delamare F., Garnier N., Robin L., 2012, « Pigments, cosmétiques ou médicaments ? Dans la tombe gallo-romaine de Saint-Médard-des-Prés (Vendée) ", in Frère D. et Hugot L. (éd.), Les huiles parfumées en Méditerranée occidentale et en Gaule (VIII ${ }^{e}$ s. av.-VI ${ }^{e}$ s. apr. J.-C.), actes du colloque Rome, École Française de Rome (16-18 nov. 2009), Rennes, Presses universitaires de Rennes, p. 191-220.

Thomas N., 2009, « L'alambic dans la cuisine ? ", in Ravoire F., Dietrich A. (éd.), La cuisine et la table dans la France de la fin du Moyen Âge, Actes $d u$ Colloque de Sens (2004), Caen, Publications du CRAHM, p. 35-50. 